Supporting Information

\title{
CRISPR-dcas9 optogenetic nanosystem for the blue light-mediated treatment of neovascular lesions
}

Jiahua Li, 1, 2, \# Yafeng Hao, 1, 2, \# Huizhuo Pan, 1, 2 Yingying Zhang, 1, 2 Guohui Cheng , 1, 2 Baona Liu, ${ }^{1,2}$ Jin Chang, ${ }^{1,2}$ Hanjie Wang, ${ }^{1,2, *}$

1 School of Life Sciences, Tianjin University, Tianjin, 300072, P.R China.

2 Tianjin Engineering Center of Micro-Nano Biomaterials and Detection-Treatment Technology, Tianjin Key Laboratory of Function and Application of Biological Macromolecular Structures, Tianjin, 300072, P.R China.

\# These authors contributed equally

* Corresponding author: Hanjie Wang (wanghj@tju.edu.cn) 


\section{Green Channel Red Channel Red Channel Merge}
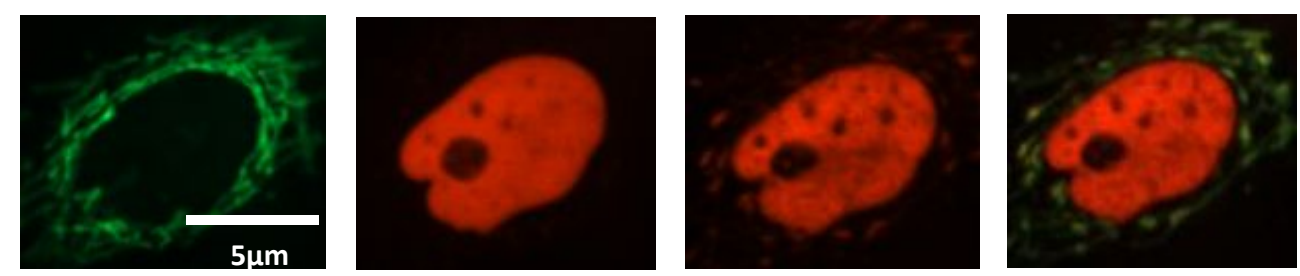

\section{Dark}

\section{Light}

(Figure S1) Light-activated translocation of cry2 in mammalian cells. After the cry2-mCherry-NLS (located in the cell nucleus) and cib1-EGFP-Tom20 (located in the mitochondria) plasmids (2:1 ratio) were cotransfection in HeLa cells using transfection reagent for $48 \mathrm{~h}$, they were irradiated using blue light ( $488 \mathrm{~nm}, 4 \mathrm{~W}$ ). The EGFP and mCherry were observed at various time points under confocal microscopy, through a 100 $\times$ oil immersion objective. 


\section{Pulsed Light}

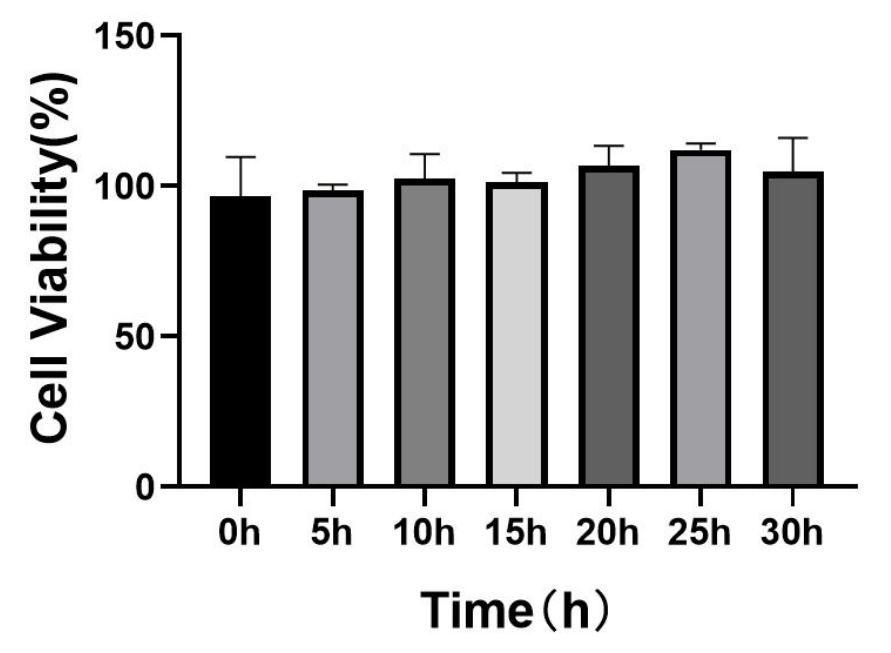

\section{Continuous Light}

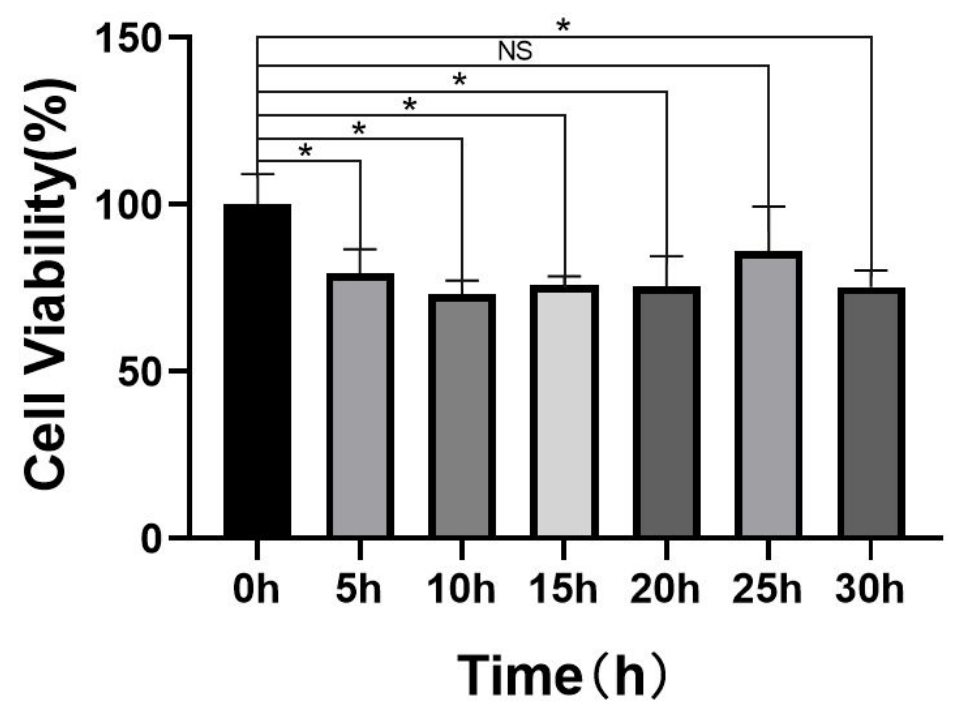

(Figure S2) The effect of plused blue-light and continuous blue-light on cell viability. mRMECs cells was treated with different light time through the plused LED array $(488 \mathrm{~nm}$ at $0.02 \mathrm{mWmm}-2$, with the illuminating frequency of $15 \mathrm{~s}$ every $1 \mathrm{~min})$ and same power continuous blue light. The MTT experimental results showed that light has less effect on cell viability under pluse light than continuous light. We used 96-well plates for MTT experiments, the number of mRMECs per well is $4 \times 10^{4} .{ }^{*}$, $p<0.05$. 


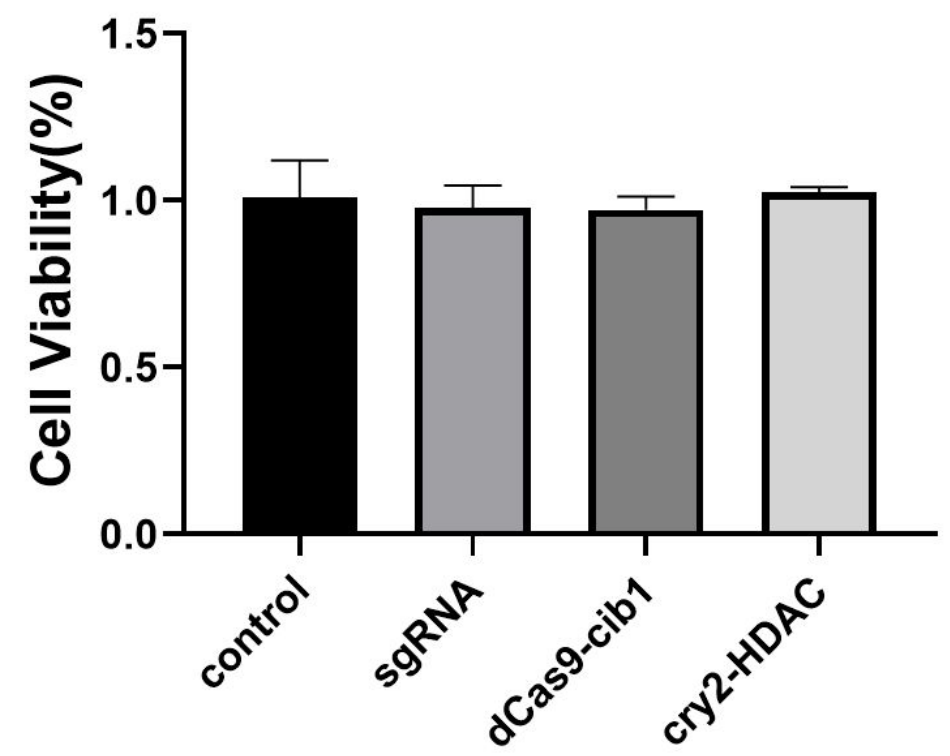

(Figure S3) Effects of plasmids overexpression on cell viability. In this opto-CRISPR system, mRMECs cells were transfected with plasmids. The MTT experimental results showed that sgRNA, dCas9-cib1 and cry2-HDAC overexpressed in these cells has no significant effect on cell viability. 


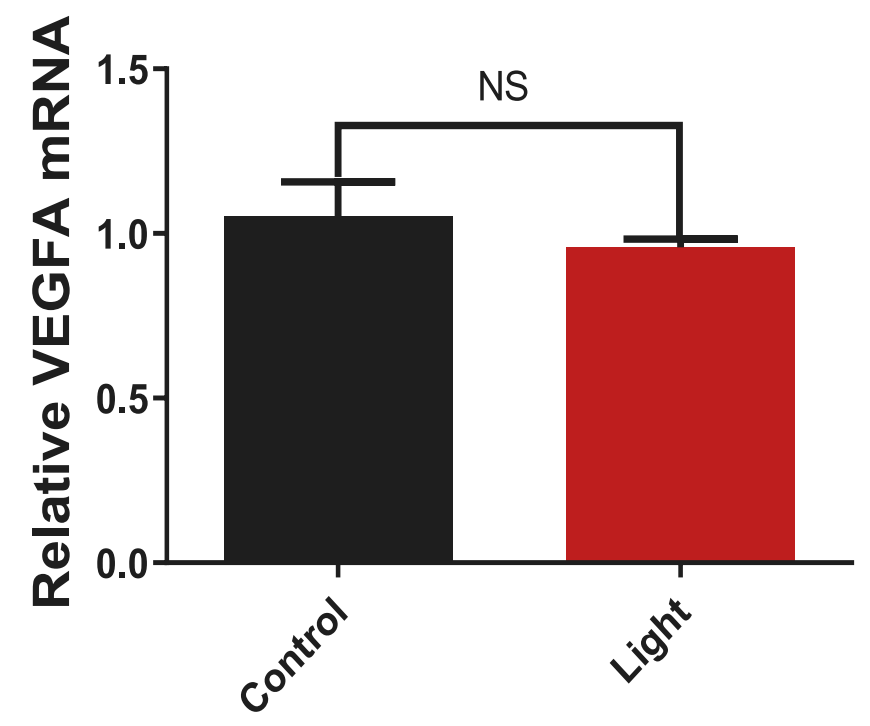

(Figure S4) Effect of blue light on VEGFA transcription. Characterization of VEGFA RNA in control group and blue light group by qPCR. In the Light group, the system was applied with blue light (488 nm at $0.02 \mathrm{mWmm}-2$ with the illuminating frequency of 15 s every $1 \mathrm{~min})$. The control group was compared with normal cell culture conditions. The test results showed that single light hardly affects VEGF expression. 


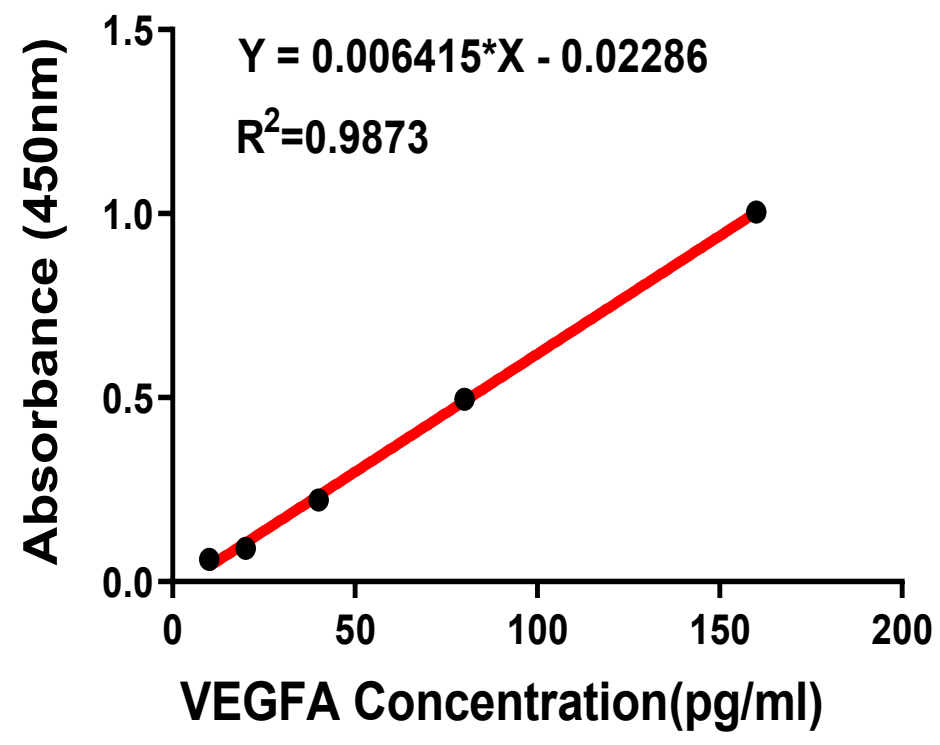

(Figure S5) Standard curve of VEGFA protein. The standard curve is determined by the VEGFA ELISA kit at $450 \mathrm{~nm}$. 


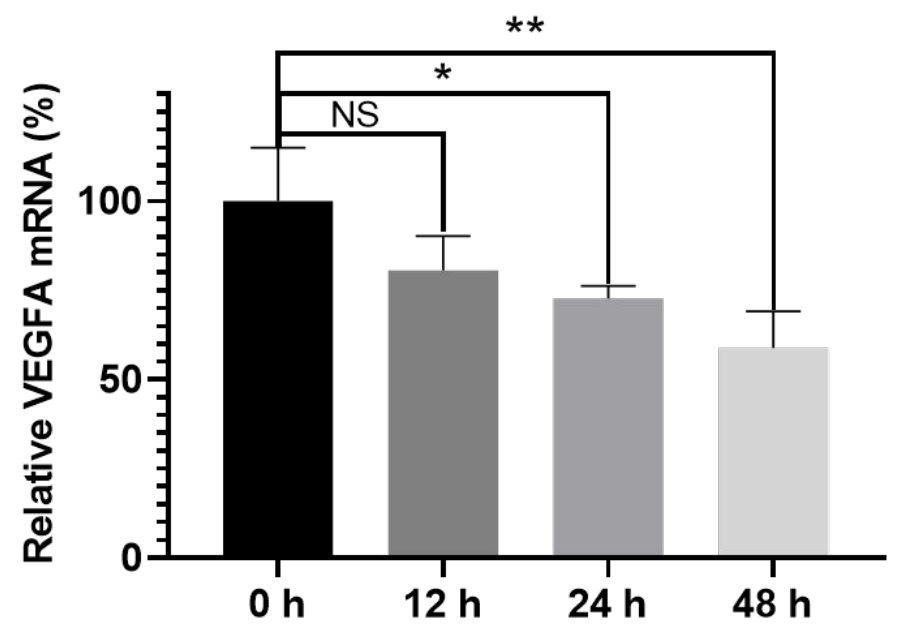

(Figure S6) The regulation effect of different lighting time on VEGF. Characterization of VEGFA in different lighting time by ELISA. The cells were irradiated for $0 \mathrm{~h}, 12 \mathrm{~h}, 24 \mathrm{~h}$ and $48 \mathrm{~h}$ hours respectively with $0.02 \mathrm{mWmm}-2$ LED (488 $\mathrm{nm}$, illuminating $15 \mathrm{~s}$ every $1 \mathrm{~min})$. The test results showed that we can control the degree of gene transcription inhibition by controlling the lighting time. ${ }^{* *}, \mathrm{p}<0.01, *$, $\mathrm{p}<0.05$. 


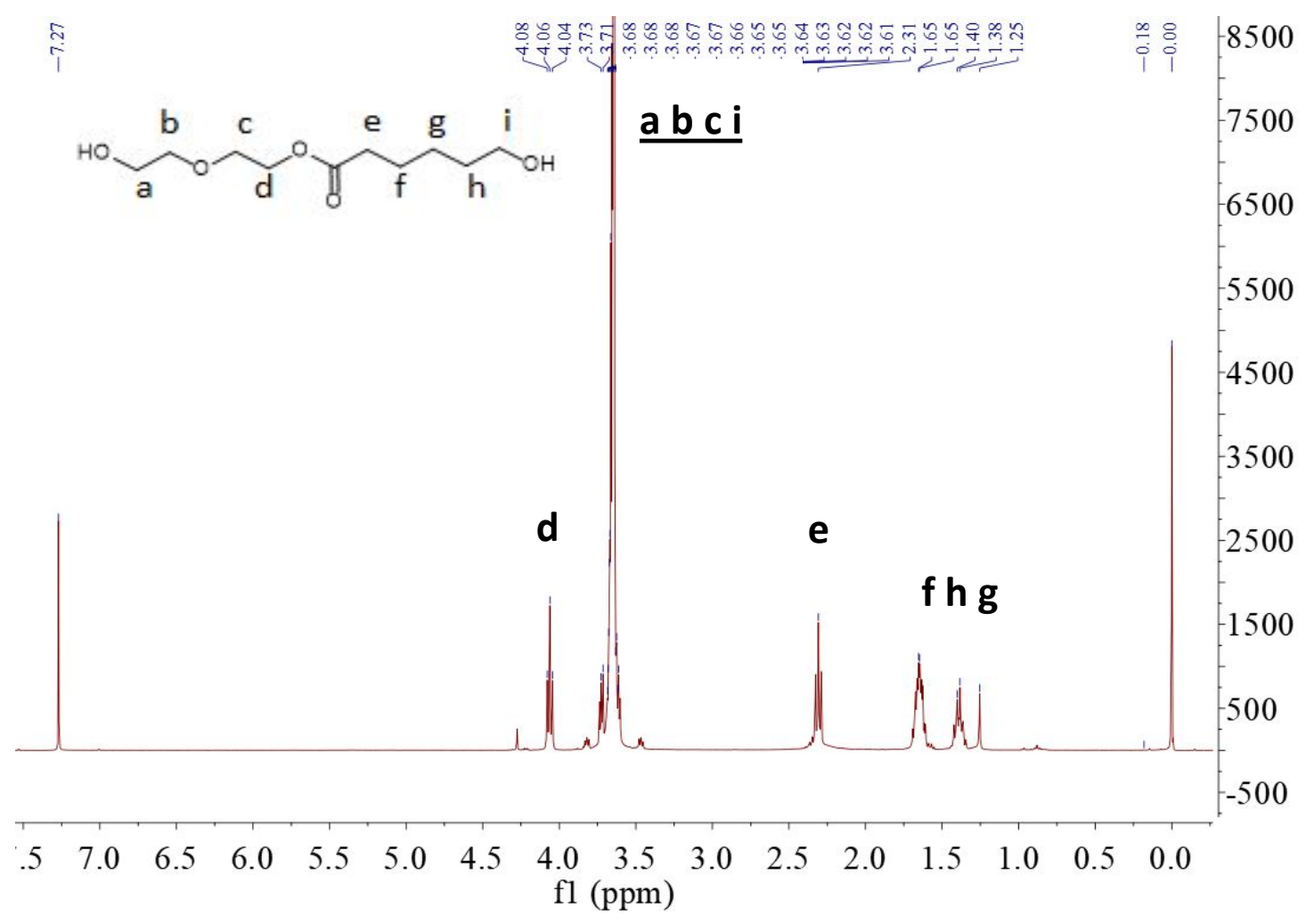

(Figure S7) Characterization of PCL-PEG chain polymer by nuclear magnetic resonance $\left({ }^{1} \mathrm{H}\right.$ NMR). 


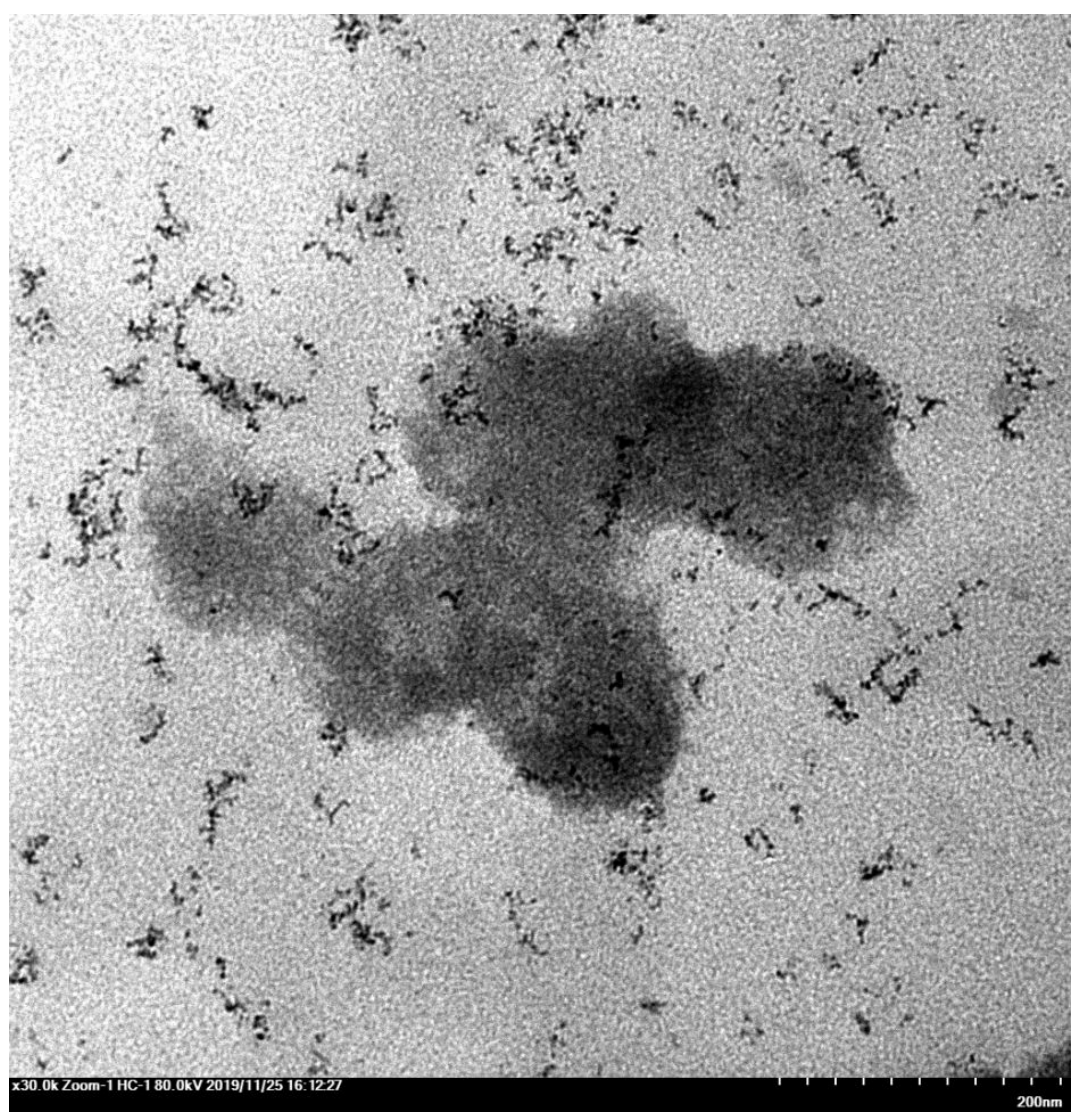

(Figure S8) TEM electron microscopy characterization of PCL-PEG nanoparticles. (scale bar: $200 \mathrm{~nm}$ ). 


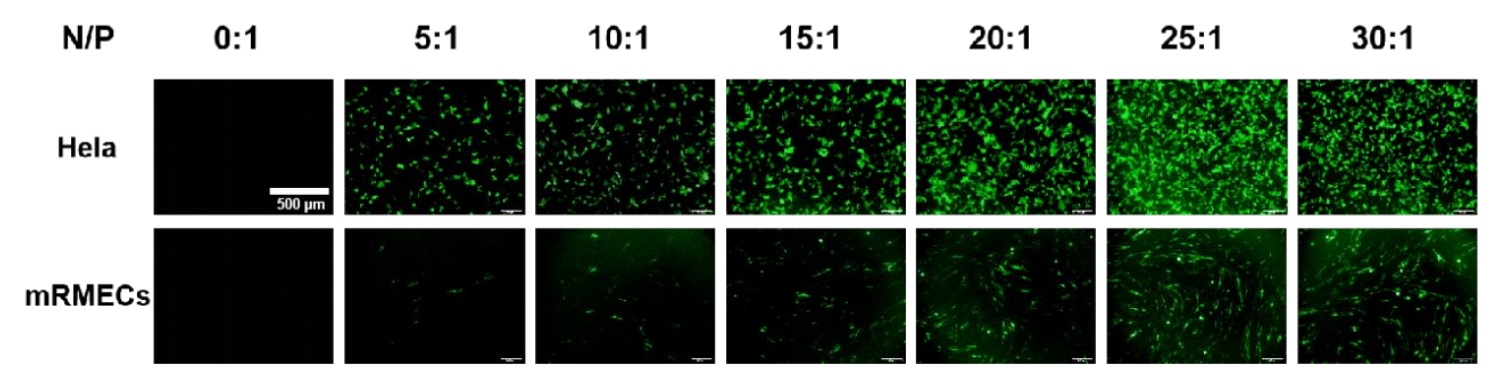

(Figure S9) Expression efficiency of GFP plasmid under different N/P ratio after

delivery. The $\mathrm{N} / \mathrm{P}$ ratio is the abbreviation of nanocarriers/plasmid. $\mathrm{N} / \mathrm{P}$ ratio: $0: 1,5: 1$, $10: 1,15: 1,20: 1,25: 1,30: 1$. 


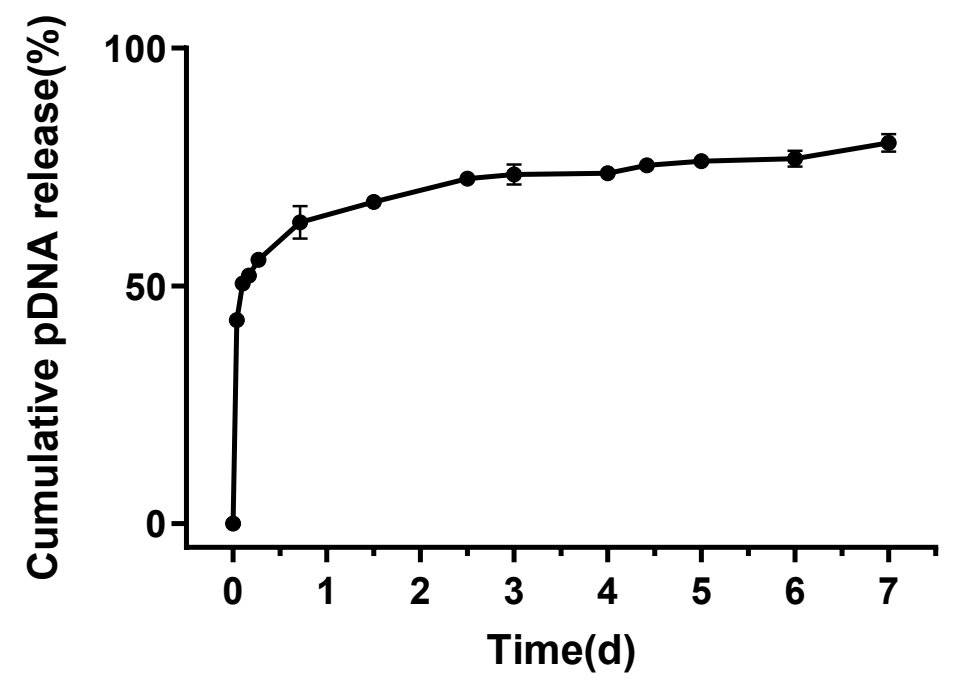

(Figure S10) In vitro plasmid release experiment. Simulate the eye environment for pDNA release experiment. Measure the absorbance at $260 \mathrm{~nm}$ by NanoDrop to calculate the pDNA concentration. 


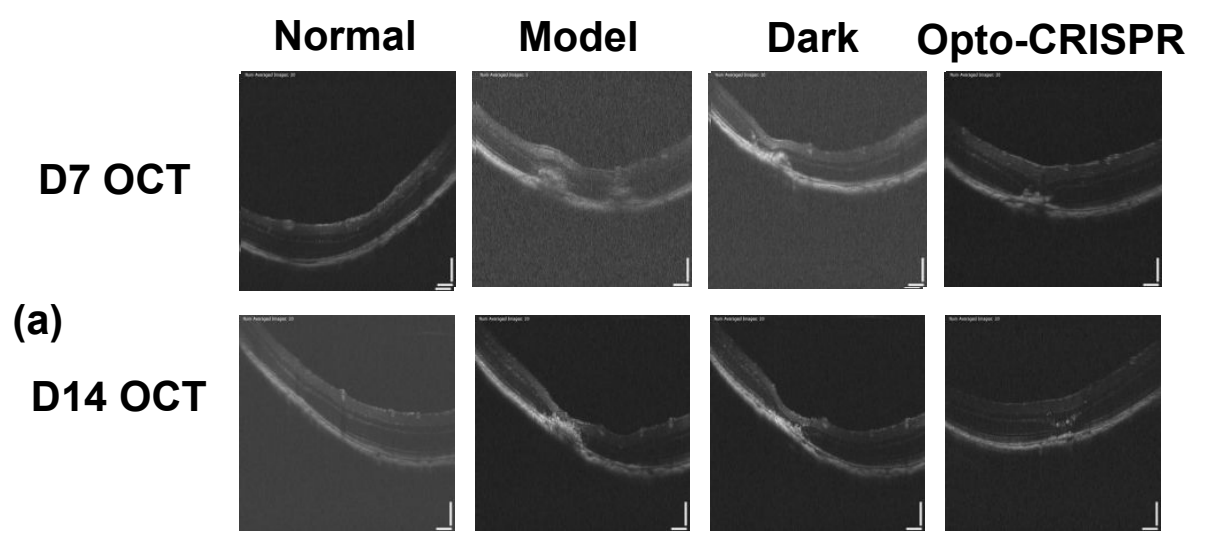

(b)

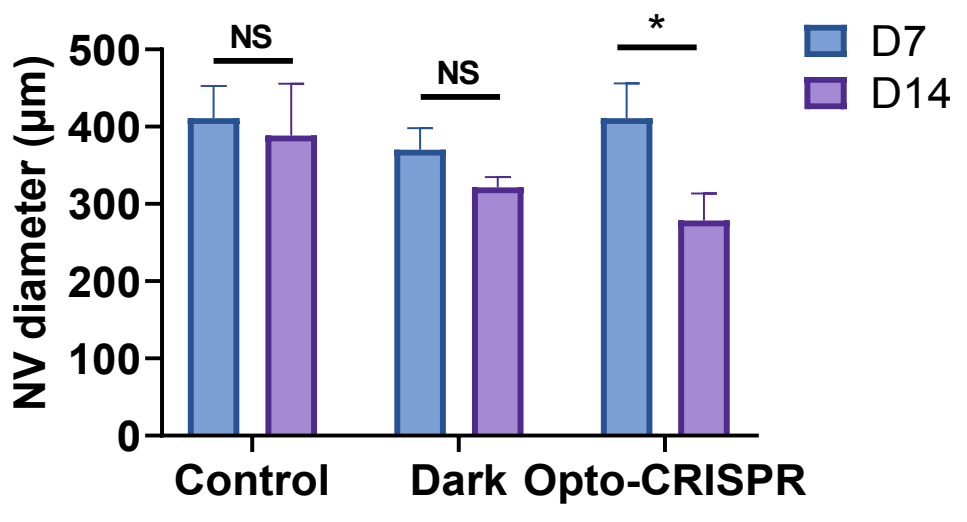


(Figure S11) Comparison of NV diameter with OCT in each group. a) Comparison of retinal structure with OCT in the control, dark and opto-CRISPR group before (D7) and after treatment (D14). b) Comparison of NV diameter with OCT in the control, dark and opto-CRISPR group before (D7) and after treatment (D14). 


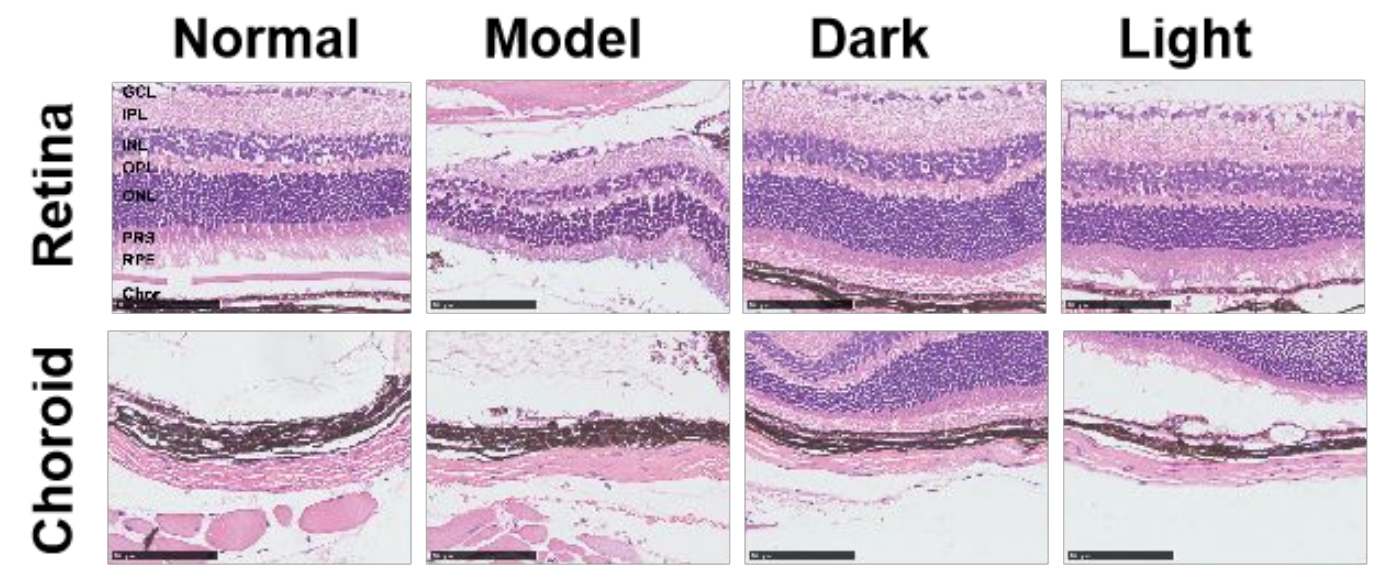

(Figure S12) Therapeutic toxicity analysis of retina and choroid using histological analysis after injection of the treatment system in each group. It shows that the injection of nanoparticles and light has no obvious toxicity to ocular tissues. The above blank control groups were injected with the same dose of blank nanoparticles. 


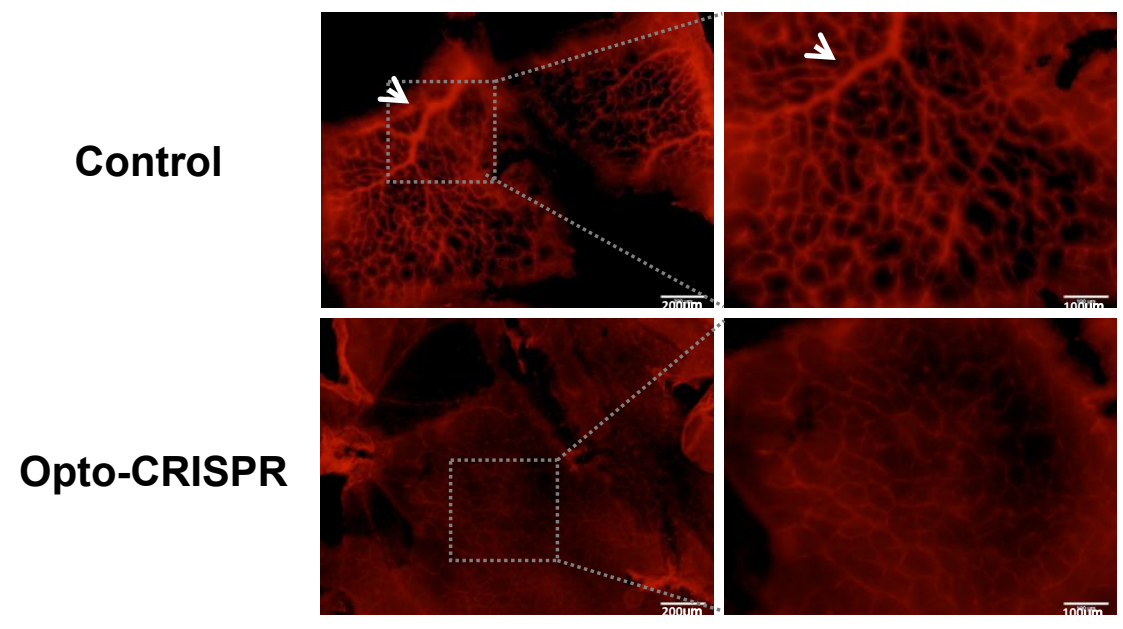

(Figure S13) Retinal blood vessel characterization in control group and dual treatment group. Retina was stained by Evans Blue dye liquor. 


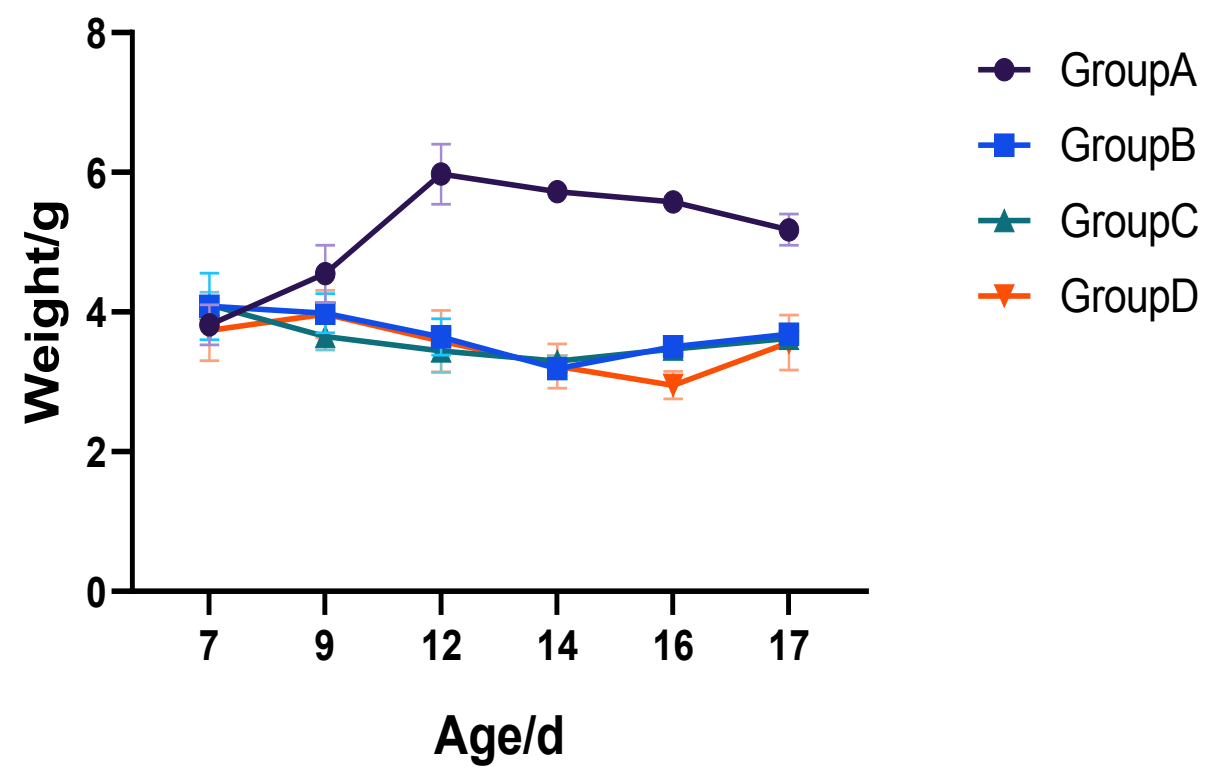

(Figure S14) Body weight of different groups of mice in different days. Group A-D: Normal group, model group, dark group, opto-CRISPR group 


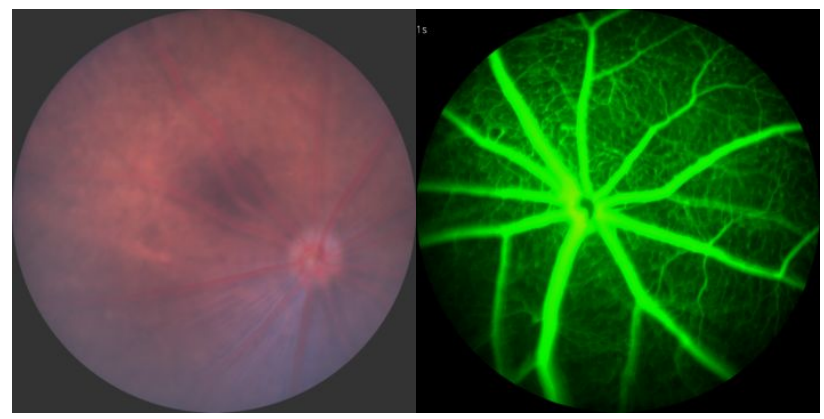

(a)

(b)

(Figure S15) Murine ocular tissue reaction to $488 \mathrm{~nm}$ LED irradiation for $30 \mathrm{~min}$. a) Fundus Photo showed clear retina with no abnormalities. b) Fluorescein imaging showed fluorescein angiography (FA) images of retinal blood vessels. 


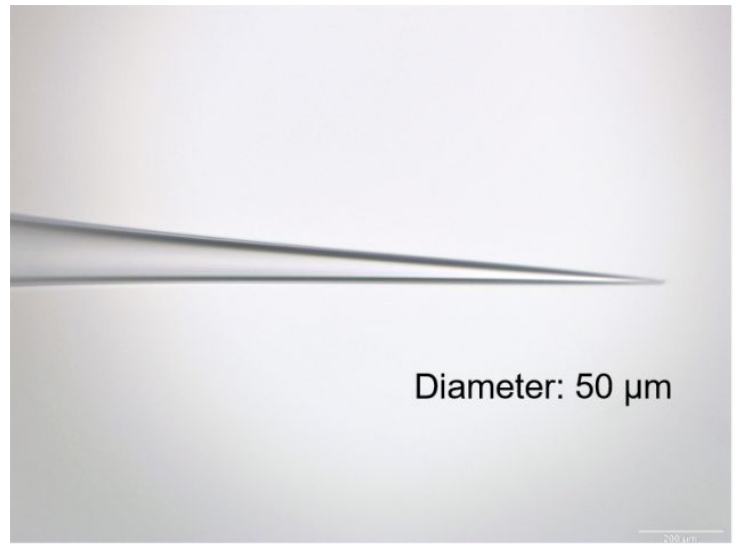

(Figure S16) Glass microelectrode for mouse vitreous injection. Outer diameter: 50 $\mu \mathrm{m}$. We used the instrument to pull the glass microelectrode and install it on the micro syringe. 


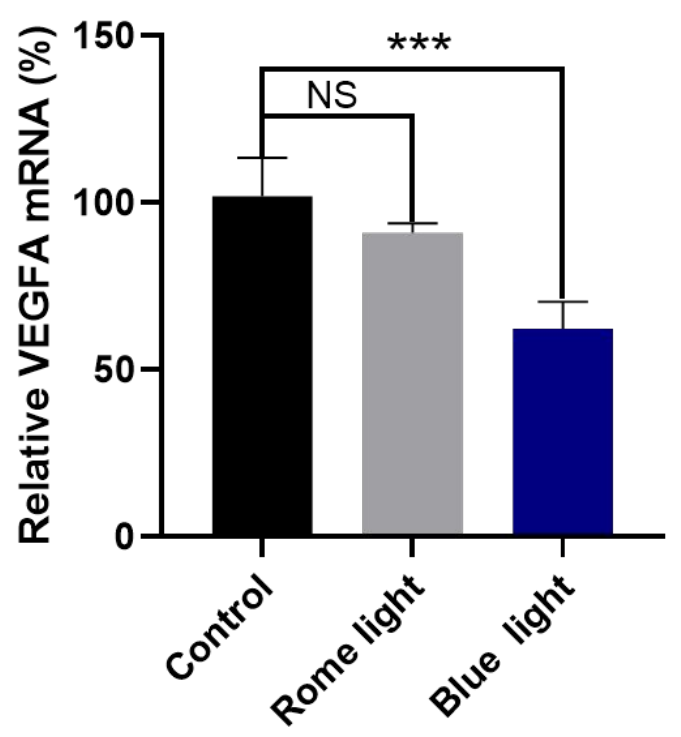

(Figure S17) The effect of the light control system being activated by different light. ELISA measurements of protein levels of VEGF transcription repression system regulated by room light and blue light. $* * *, \mathrm{p}<0.001$. 


\section{Supplementary Table S1:}

Table S1 Primers for RNA analysis and antibodies' information

\begin{tabular}{|c|c|c|}
\hline Primer name & \multicolumn{2}{|l|}{ Sequence } \\
\hline VEGF forward & \multicolumn{2}{|c|}{ 5'-CCCCGACGAGATAGAGTA-3' } \\
\hline VEGF reverse & \multicolumn{2}{|c|}{ 5'-GCTTTGGTGAGGTTTGAT-3' } \\
\hline Actin forward & \multicolumn{2}{|c|}{ 5'-GAGACCTTCAACACCCCAGC-3' } \\
\hline Actin reverse & \multicolumn{2}{|c|}{ 5'-ATGTCACGCACGATTTCCC-3' } \\
\hline Antibodies & SOURCE & IDENTIFIER \\
\hline Rabbit anti-VEGFA & Abcam & Ab1316 \\
\hline$\beta$-tublin & Thermo Fisher & MA5-16308 \\
\hline $\begin{array}{l}\text { Goat anti-Rabbit } \\
\operatorname{lgG}(\mathrm{H}+\mathrm{L})-\mathrm{HPR}\end{array}$ & SUNGENE BIOTECH & LK2001 \\
\hline $\begin{array}{l}\text { Goat anti-Mouse } \\
\operatorname{lgG}(\mathrm{H}+\mathrm{L})-\mathrm{HPR}\end{array}$ & SUNGENE BIOTECH & LK2003 \\
\hline
\end{tabular}

\section{Supplementary Note}

Sequence information:

Nucleic acid sequence of sgRNA

sgRNA1: CGACAAGGCAGACTATTCAG (Distance to TSS is +112)

sgRNA2: ACCGTTGGCACGATTTAAGA (Distance to TSS is +246) 


\section{Nucleic acid sequence of dCas9-cib1}

TTCATCTTCTTGATGCTTTTTGTGCTCCCATTGTTTTGCTCTGTTATTTTCGACT TCTCTTCCTCTCCCTCTTCTACTAGGTCATCTCTGTTCATCGTCATCTTCTTCTT CTTCTCATTACAATCCTTAGTCTCTGTATCAAACTTCCGTTTCTTGAAATTTCC AGTCCCAAGCGTCGTTTCCGGCGAAATTGAGAGTCTTGAATCACCTTCCACC GTCGTCTCACCGTACATCATCGGAAGATTCAAACCGGCAGTCGAAAGATAGC TGTCCATCTCGCCGCCGGTAATCATTGAAGAATCGGCAAAGAAGCCGGCGAG AGGAGAATCAAAGGTGGGATTGAGGTACTTGAGGTGAGCCCTTTGGCGCTCT AGGACCGACATGTCAGGAAAATTGAGCAAAAGGTCACCTCCTATAGCTCCAT TTATATCGATGTCATGATCTTTATAATCACCGTCATGGTCTTTGTAGTCCATG GCGCTAGCCAGCTTGGGTCTCCCTATAGTGAGTCGTATTAATTTCGATAAGCC AGTAAGCAGTGGGTTCTCTAGTTAGCCAGAGAGCTCTGCTTATATAGACCTC CCACCGTACACGCCTACCGCCCATTTGCGTCAATGGGGCGGAGTTGTTACGA CATTTTGGAAAGTCCCGTTGATTTTGGTGCCAAAACAAACTCCCATTGACGTC AATGGGGTGGAGACTTGGAAATCCCCGTGAGTCAAACCGCTATCCACGCCCA TTGATGTACTGCCAAAACCGCATCACCATGGTAATAGCGATGACTAATACGT AGATGTACTGCCAAGTAGGAAAGTCCCATAAGGTCATGTACTGGGCATAATG CCAGGCGGGCCATTTACCGTCATTGACGTCAATAGGGGGCGTACTTGGCATA TGATACACTTGATGTACTGCCAAGTGGGCAGTTTACCGTAAATACTCCACCC ATTGACGTCAATGGAAAGTCCCTATTGGCGTTACTATGGGAACATACGTCAT TATTGACGTCAATGGGCGGGGGTCGTTGGGCGGTCAGCCAGGCGGGCCATTT ACCGTAAGTTATGTAACGCGGAACTCCATATATGGGCTATGAACTAATGACC CCGTAATTGATTACTATTAATAACTAGTCAATAATCAATGTCAACGCGTATAT CTGGCCCGTACATCGCGAAGCAGCGCAAAACGCCTAACCCTAAGCAGATTCT TCATGCAATTGTCGGTCAAGCCTTGCCTTGTTGTAGCTTAAATTTTGCTCGCG CACTACTCAGCGACCTCCAACACACAAGCAGGGAGCAGATACTGGCTTAACT ATGCGGCATCAGAGCAGATTGTACTGAGAGTGCACCATAGGGGATCGGGAG ATCTCCCGATCCGTCGACGTCAGGTGGCACTTTTCGGGGAAATGTGCGCGGA ACCCCTATTTGTTTATTTTTCTAAATACATTCAAATATGTATCCGCTCATGAG ACAATAACCCTGATAAATGCTTCAATAATATTGAAAAAGGAAGAGTATGAGT ATTCAACATTTCCGTGTCGCCCTTATTCCCTTTTTTGCGGCATTTTGCCTTCCT GTTTTTGCTCACCCAGAAACGCTGGTGAAAGTAAAAGATGCTGAAGATCAGT TGGGTGCACGAGTGGGTTACATCGAACTGGATCTCAACAGCGGTAAGATCCT 
TGAGAGTTTTCGCCCCGAAGAACGTTTTCCAATGATGAGCACTTTTAAAGTTC TGCTATGTGGCGCGGTATTATCCCGTATTGACGCCGGGCAAGAGCAACTCGG TCGCCGCATACACTATTCTCAGAATGACTTGGTTGAGTACTCACCAGTCACA GAAAAGCATCTTACGGATGGCATGACAGTAAGAGAATTATGCAGTGCTGCC ATAACCATGAGTGATAACACTGCGGCCAACTTACTTCTGACAACGATCGGAG GACCGAAGGAGCTAACCGCTTTTTTGCACAACATGGGGGATCATGTAACTCG CCTTGATCGTTGGGAACCGGAGCTGAATGAAGCCATACCAAACGACGAGCG TGACACCACGATGCCTGTAGCAATGGCAACAACGTTGCGCAAACTATTAACT GGCGAACTACTTACTCTAGCTTCCCGGCAACAATTAATAGACTGGATGGAGG CGGATAAAGTTGCAGGACCACTTCTGCGCTCGGCCCTTCCGGCTGGCTGGTT TATTGCTGATAAATCTGGAGCCGGTGAGCGTGGGTCTCGCGGTATCATTGCA GCACTGGGGCCAGATGGTAAGCCCTCCCGTATCGTAGTTATCTACACGACGG GGAGTCAGGCAACTATGGATGAACGAAATAGACAGATCGCTGAGATAGGTG CCTCACTGATTAAGCATTGGTAACTGTCAGACCAAGTTTACTCATATATACTT TAGATTGATTTAAAACTTCATTTTTAATTTAAAAGGATCTAGGTGAAGATCCT TTTTGATAATCTCATGACCAAAATCCCTTAACGTGAGTTTTCGTTCCACTGAG CGTCAGACCCCGTAGAAAAGATCAAAGGATCTTCTTGAGATCCTTTTTTTCTG CGCGTAATCTGCTGCTTGCAAACAAAAAAACCACCGCTACCAGCGGTGGTTT GTTTGCCGGATCAAGAGCTACCAACTCTTTTTCCGAAGGTAACTGGCTTCAG CAGAGCGCAGATACCAAATACTGTTCTTCTAGTGTAGCCGTAGTTAGGCCAC CACTTCAAGAACTCTGTAGCACCGCCTACATACCTCGCTCTGCTAATCCTGTT ACCAGTGGCTGCTGCCAGTGGCGATAAGTCGTGTCTTACCGGGTTGGACTCA AGACGATAGTTACCGGATAAGGCGCAGCGGTCGGGCTGAACGGGGGGTTCG TGCACACAGCCCAGCTTGGAGCGAACGACCTACACCGAACTGAGATACCTAC AGCGTGAGCTATGAGAAAGCGCCACGCTTCCCGAAGGGAGAAAGGCGGACA GGTATCCGGTAAGCGGCAGGGTCGGAACAGGAGAGCGCACGAGGGAGCTTC CAGGGGGAAACGCCTGGTATCTTTATAGTCCTGTCGGGTTTCGCCACCTCTG ACTTGAGCGTCGATTTTTGTGATGCTCGTCAGGGGGGCGGAGCCTATGGAAA AACGCCAGCAACGCGGCCTTTTTACGGTTCCTGGCCTTTTGCTGGCCTTTTGC TCACATGTTCTTTCCTGCGTTATCCCCTGATTCTGTGGATAACCGTATTACCG CCTTTGAGTGAGCTGATACCGCTCGCCGCAGCCGAACGACCGAGCGCAGCGA GTCAGTGAGCGAGGAAGCGGAAGAGCGCCCAATACGCAAACCGCCTCTCCC CGCGCGTTGGCCGATTCATTAATGCAGCTGGCACGACAGGTTTCCCGACTGG AAAGCGGGCAGTGAGCGCAACGCAATTAATGTGAGTTAGCTCACTCATTAGG CACCCCAGGCTTTACACTTTATGCTTCCGGCTCGTATGTTGTGTGGAATTGTG AGCGGATAACAATTTCACACAGGAAACAGCTATGACCATGATTACGCCAAG 
CTCTAGCTAGAGGTCGACGGTATACAGACATGATAAGATACATTGATGAGTT TGGACAAACCACAACTAGAATGCAGTGAAAAAAATGCTTTATTTGTGAAATT TGTGATGCTATTGCTTTATTTGTAACCATTATAAGCTGCAATAAACAAGTTGG GGTGGGCGAAGAACTCCAGCATGAGATCCCCGCGCTGGAGGATCATCCAGC CGGCGTCCCGGAAAACGATTCCGAAGCCCAACCTTTCATAGAAGGCGGCGGT GGAATCGAAATCTCGTGATGGCAGGTTGGGCGTCGCTTGGTCGGTCATTTCG AACCCCAGAGTCCCGCTCAGAAGAACTCGTCAAGAAGGCGATAGAAGGCGA TGCGCTGCGAATCGGGAGCGGCGATACCGTAAAGCACGAGGAAGCGGTCAG CCCATTCGCCGCCAAGCTCTTCAGCAATATCACGGGTAGCCAACGCTATGTC CTGATAGCGGTCCGCCACACCCAGCCGGCCACAGTCGATGAATCCAGAAAA GCGGCCATTTTCCACCATGATATTCGGCAAGCAGGCATCGCCATGGGTCACG ACGAGATCCTCGCCGTCGGGCATGCGCGCCTTGAGCCTGGCGAACAGTTCGG CTGGCGCGAGCCCCTGATGCTCTTCGTCCAGATCATCCTGATCGACAAGACC GGCTTCCATCCGAGTACGTGCTCGCTCGATGCGATGTTTCGCTTGGTGGTCGA ATGGGCAGGTAGCCGGATCAAGCGTATGCAGCCGCCGCATTGCATCAGCCAT GATGGATACTTTCTCGGCAGGAGCAAGGTGAGATGACAGGAGATCCTGCCCC GGCACTTCGCCCAATAGCAGCCAGTCCCTTCCCGCTTCAGTGACAACGTCGA GCACAGCTGCGCAAGGAACGCCCGTCGTGGCCAGCCACGATAGCCGCGCTG CCTCGTCCTGCAGTTCATTCAGGGCACCGGACAGGTCGGTCTTGACAAAAAG AACCGGGCGCCCCTGCGCTGACAGCCGGAACACGGCGGCATCAGAGCAGCC GATTGTCTGTTGTGCCCAGTCATAGCCGAATAGCCTCTCCACCCAAGCGGCC GGAGAACCTGCGTGCAATCCATCTTGTTCAATCATGCGAAACGATCCTCATC CTGTCTCTTGATCAGATCCGAAAATGGATATACAAGCTCCCGGGAGCTTTTT GCAAAAGCCTAGGCCTCCAAAAAAGCCTCCTCACTACTTCTGGAATAGCTCA GAGGCAGAGGCGGCCTCGGCCTCTGCATAAATAAAAAAAATTAGTCAGCCA TGGGGCGGAGAATGGGCGGAACTGGGCGGAGTTAGGGGCGGGATGGGCGG AGTTAGGGGCGGGACTATGGTTGCTGACTAATTGAGATGCATGCTTTGCATA CTTCTGCCTGCTGGGGAGCCTGGGGACTTTCCACACCTGGTTGCTGACTAATT GAGATGCATGCTTTGCATACTTCTGCCTGCTGGGGAGCCTGGGGACTTTCCA CACCCTAACTGACACACATTCCACAGAATTAATTCGCGTTAAATTTTTGTTAA ATCAGCTCATTTTTTAACCAATAGGCCGAAATCGGCAAAATCCCTTATAAAT CAAAAGAATAGACCGAGATAGGGTTGAGTGTTGTTCCAGTTTGGAACAAGA GTCCACTATTAAAGAACGTGGACTCCAACGTCAAAGGGCGAAAAACCGTCT ATCAGGGCGATGGCCCACTACGTGAACCATCACCCTAATCAAGTTTTTTGGG GTCGAGGTGCCGTAAAGCACTAAATCGGAACCCTAAAGGGAGCCCCCGATTT AGAGCTTGACGGGGAAAGCCGGCGAACGTGGCGAGAAAGGAAGGGAAGAA 
AGCGAAAGGAGCGGGCGCTAGGGCGCTGGCAAGTGTAGCGGTCACGCTGCG CGTAACCACCACACCCGCCGCGCTTAATGCGCCGCTACAGGGCGCGTGGGGA TACCCCCTAGAGCCCCAGCTGGTTCTTTCCGCCTCAGAAGCCATAGAGCCCA CCGCATCCCCAGCATGCCTGCTATTGTCTTCCCAATCCTCCCCCTTGCTGTCC TGCCCCACCCCACCCCCCAGAATAGAATGACACCTACTCAGACAATGCGATG CAATTTCCTCATTTTATTAGGAAAGGACAGTGGGAGTGGCACCTTCCAGGGT CAAGGAAGGCACGGGGGAGGGGCAAACAACAGATGGCTGGCAACTAGAAA GGCACAGTCGAGGCTTGATCAGCGGTTTAAACTTAAGTTTAAATATAATCCG TTTTCTCCAATTCCTTCGTCACTTTAGATGAATCATTAGAGAAATTGTTCTCTT CTTTCTTGGCTTGGGGTTTCATCTTCTTGATGCTTTTTGTGCTCCCATTGTTTG GCTCTGTTATTTTCCGACTTCTCTTCTTCTCCTTCTTCAACTAAGGCATCTCTG TTCATCGTCATCTTCTTTTTTCTTGCCCATTAAAAAACCT

\section{Nucleic acid sequence of cry2-HDAC}

GACGGATCGGGAGATCTCCCGATCCCCTATGGTGCACTCTCAGTACAATCTG CTCTGATGCCGCATAGTTAAGCCAGTATCTGCTCCCTGCTTGTGTGTTGGAGG TCGCTGAGTAGTGCGCGAGCAAAATTTAAGCTACAACAAGGCAAGGCTTGA CCGACAATTGCATGAAGAATCTGCTTAGGGTTAGGCGTTTTGCGCTGCTTCG CGATGTACGGGCCAGATATACGCGTTGACATTGATTATTGACTAGTTATTAA TAGTAATCAATTACGGGGTCATTAGTTCATAGCCCATATATGGAGTTCCGCG TTACATAACTTACGGTAAATGGCCCGCCTGGCTGACCGCCCAACGACCCCCG CCCATTGACGTCAATAATGACGTATGTTCCCATAGTAACGCCAATAGGGACT TTCCATTGACGTCAATGGGTGGAGTATTTACGGTAAACTGCCCACTTGGCAG TACATCAAGTGTATCATATGCCAAGTACGCCCCCTATTGACGTCAATGACGG TAAATGGCCCGCCTGGCATTATGCCCAGTACATGACCTTATGGGACTTTCCTA CTTGGCAGTACATCTACGTATTAGTCATCGCTATTACCATGGTGATGCGGTTT TGGCAGTACATCAATGGGCGTGGATAGCGGTTTGACTCACGGGGATTTCCAA GTCTCCACCCCATTGACGTCAATGGGAGTTTGTTTTGGCACCAAAATCAACG GGACTTTCCAAAATGTCGTAACAACTCCGCCCCATTGACGCAAATGGGCGGT AGGCGTGTACGGTGGGAGGTCTATATAAGCAGAGCTCTCTGGCTAACTAGAG AACCCACTGCTTACTGGCTTATCGAAATtagaacgagctggtgcacgtggettcccaaagatctctca gataatgagaggaaatgcagtcatcagtttgcagaaggctagggattctgggccATAGCTCAGACCTGCGCC CACCATCTCCCTCCAGGCAGCCCTTGGGAGACCCAAGCTGGCTAGCGTTTAA ACTTAAGCTTGATGCCTAAGAAAAAGAGGAAGGTGAAGATGGACAAAAAGA 
CTATAGTTTGGTTTAGAAGAGACCTAAGGATTGAGGATAATCCTGCATTAGC AGCAGCTGCTCACGAAGGATCTGTTTTTCCTGTCTTCATTTGGTGTCCTGAAG AAGAAGGACAGTTTTATCCTGGAAGAGCTTCAAGATGGTGGATGAAACAAT CACTTGCTCACTTATCTCAATCCTTGAAGGCTCTTGGATCTGACCTCACTTTA ATCAAAACCCACAACACGATTTCAGCGATCTTGGATTGTATCCGCGTTACCG GTGCTACAAAAGTCGTCTTTAACCACCTCTATGATCCTGTTTCGTTAGTTCGG GACCATACCGTAAAGGAGAAGCTGGTGGAACGTGGGATCTCTGTGCAAAGC TACAATGGAGATCTATTGTATGAACCGTGGGAGATATACTGCGAAAAGGGC AAACCTTTTACGAGTTTCAATTCTTACTGGAAGAAATGCTTAGATATGTCGAT TGAATCCGTTATGCTTCCTCCTCCTTGGCGGTTGATGCCAATAACTGCAGCGG CTGAAGCGATTTGGGCGTGTTCGATTGAAGAACTAGGGCTGGAGAATGAGG CCGAGAAACCGAGCAATGCGTTGTTAACTAGAGCTTGGTCTCCAGGATGGAG CAATGCTGATAAGTTACTAAATGAGTTCATCGAGAAGCAGTTGATAGATTAT GCAAAGAACAGCAAGAAAGTTGTTGGGAATTCTACTTCACTACTTTCTCCGT ATCTCCATTTCGGGGAAATAAGCGTCAGACACGTTTTCCAGTGTGCCCGGAT GAAACAAATTATATGGGCAAGAGATAAGAACAGTGAAGGAGAAGAAAGTG CAGATCTTTTTCTTAGGGGAATCGGTTTAAGAGAGTATTCTCGGTATATATGT TTCAACTTCCCGTTTACTCACGAGCAATCGTTGTTGAGTCATCTTCGGTTTTTC CCTTGGGATGCTGATGTTGATAAGTTCAAGGCCTGGAGACAAGGCAGGACCG GTTATCCGTTGGTGGATGCCGGAATGAGAGAGCTTTGGGCTACCGGATGGAT GCATAACAGAATAAGAGTGATTGTTTCAAGCTTTGCTGTGAAGTTTCTTCTCC TTCCATGGAAATGGGGAATGAAGTATTTCTGGGATACACTTTTGGATGCTGA TTTGGAATGTGACATCCTTGGCTGGCAGTATATCTCTGGGAGTATCCCCGATG GCCACGAGCTTGATCGCTTGGACAATCCCGCGTTACAAGGCGCCAAATATGA CCCAGAAGGTGAGTACATAAGGCAATGGCTTCCCGAGCTTGCGAGATTGCCA ACTGAATGGATCCATCATCCATGGGACGCTCCTTTAACCGTACTCAAAGCTT CTGGTGTGGAACTCGGAACAAACTATGCGAAACCCATTGTAGACATCGACAC AGCTCGTGAGCTACTAGCTAAAGCTATTTCAAGAACCCGTGAAGCACAGATC ATGATCGGAGCAGCAGCAGGAGGAGGTGGAAGCGGAGGAGGAGGTAGCgcca agaccgtggcgtatttctacgacccegatgtgggcaacttccactatggagctggacacccaatgaaacctcatcgectggcatt gactcatagcctagtcctgcattatggtctctataagaagatgatcgtcttcaagcettaccaggcctccagcatgacatgtgec gcttccattctgaggactacatcgacttcctgcagagagtcagccccaccaatatgcagggtttcaccaagagccttaatgccttc aacgtgggtgatgactgccagtgtttccaggacttttcgagttctgctcccgttacacaggcgcatctctgcaaggagcaacac agctaaacaacaagatctgtgatattgccatcaactgggecggtggtctacatcatgccaagaaatttgaggcctctggcttctgc tatgtcaatgacatagtaattggtatcctggagctgcttaagtaccaccetcgggtgctctacattgatatcgacatccaccatggt gacggggttcaggaagcettctacctcactgaccgggtcatgactgtgtccttccacaaatacggaaattacttctttcctggaac 
aggtgacatgtatgaagttggagcagagagtggccgctactattgtctcaatgtgccettacgagatggcattgatgaccagagt tacaagcaccttttccagccagtcatcagccaggtggtggacttctaccagccgacgtgcatcgtgctccagtgtggegctgact ccctgggetgtgatcgattaggctgcttcaatctcagcattcgaggacatggggaatgtgttgaatatgtcaagagtttcaatatcc ctctcctggtactgggaggtggtggctacactgtccgaaatgttgcccggtgttggacatatgaaacatctctgctggtagaaga ggccattagtgaggaacttccctatagtgaatacttcgagtactttgccccagatttcacactccatccagatgtcagcacccgcat cgagaatcagaactcacgccagtatctggaccagatccgccagacaatctttgaaaacttgaagatgctgaaccatgcacccag tgtccagattcatgatgtcccggcagacctcctgacgtatgacaggactgacgaggccgacgctgaagagagaggtcccgag gagaactacagcaggccagaagcacccaatgagttctatgatggcgaccatgacaacgacaaggaaagtgatgtggagattt agTTTAAACCCGCTGATCAGCCTCGACTGTGCCTTCTAGTTGCCAGCCATCTGT TGTTTGCCCCTCCCCCGTGCCTTCCTTGACCCTGGAAGGTGCCACTCCCACTG TCCTTTCCTAATAAAATGAGGAAATTGCATCGCATTGTCTGAGTAGGTGTCAT TCTATTCTGGGGGGTGGGGTGGGGCAGGACAGCAAGGGGGAGGATTGGGAA GACAATAGCAGGCATGCTGGGGATGCGGTGGGCTCTATGGCTTCTGAGGCGG AAAGAACCAGCTGGGGCTCTAGGGGGTATCCCCACGCGCCCTGTAGCGGCG CATTAAGCGCGGCGGGTGTGGTGGTTACGCGCAGCGTGACCGCTACACTTGC CAGCGCCCTAGCGCCCGCTCCTTTCGCTTTCTTCCCTTCCTTTCTCGCCACGTT CGCCGGCTTTCCCCGTCAAGCTCTAAATCGGGGGCTCCCTTTAGGGTTCCGAT TTAGTGCTTTACGGCACCTCGACCCCAAAAAACTTGATTAGGGTGATGGTTC ACGTAGTGGGCCATCGCCCTGATAGACGGTTTTTCGCCCTTTGACGTTGGAGT CCACGTTCTTTAATAGTGGACTCTTGTTCCAAACTGGAACAACACTCAACCCT ATCTCGGTCTATTCTTTTGATTTATAAGGGATTTTGCCGATTTCGGCCTATTG GTTAAAAAATGAGCTGATTTAACAAAAATTTAACGCGAATTAATTCTGTGGA ATGTGTGTCAGTTAGGGTGTGGAAAGTCCCCAGGCTCCCCAGCAGGCAGAAG TATGCAAAGCATGCATCTCAATTAGTCAGCAACCAGGTGTGGAAAGTCCCCA GGCTCCCCAGCAGGCAGAAGTATGCAAAGCATGCATCTCAATTAGTCAGCAA CCATAGTCCCGCCCCTAACTCCGCCCATCCCGCCCCTAACTCCGCCCAGTTCC GCCCATTCTCCGCCCCATGGCTGACTAATTTTTTTTATTTATGCAGAGGCCGA GGCCGCCTCTGCCTCTGAGCTATTCCAGAAGTAGTGAGGAGGCTTTTTTGGA GGCCTAGGCTTTTGCAAAAAGCTCCCGGGAGCTTGTATATCCATTTTCGGATC TGATCAAGAGACAGGATGAGGATCGTTTCGCATGATTGAACAAGATGGATTG CACGCAGGTTCTCCGGCCGCTTGGGTGGAGAGGCTATTCGGCTATGACTGGG CACAACAGACAATCGGCTGCTCTGATGCCGCCGTGTTCCGGCTGTCAGCGCA GGGGCGCCCGGTTCTTTTTGTCAAGACCGACCTGTCCGGTGCCCTGAATGAA CTGCAGGACGAGGCAGCGCGGCTATCGTGGCTGGCCACGACGGGCGTTCCTT GCGCAGCTGTGCTCGACGTTGTCACTGAAGCGGGAAGGGACTGGCTGCTATT GGGCGAAGTGCCGGGGCAGGATCTCCTGTCATCTCACCTTGCTCCTGCCGAG 
AAAGTATCCATCATGGCTGATGCAATGCGGCGGCTGCATACGCTTGATCCGG CTACCTGCCCATTCGACCACCAAGCGAAACATCGCATCGAGCGAGCACGTAC TCGGATGGAAGCCGGTCTTGTCGATCAGGATGATCTGGACGAAGAGCATCAG GGGCTCGCGCCAGCCGAACTGTTCGCCAGGCTCAAGGCGCGCATGCCCGACG GCGAGGATCTCGTCGTGACCCATGGCGATGCCTGCTTGCCGAATATCATGGT GGAAAATGGCCGCTTTTCTGGATTCATCGACTGTGGCCGGCTGGGTGTGGCG GACCGCTATCAGGACATAGCGTTGGCTACCCGTGATATTGCTGAAGAGCTTG GCGGCGAATGGGCTGACCGCTTCCTCGTGCTTTACGGTATCGCCGCTCCCGA TTCGCAGCGCATCGCCTTCTATCGCCTTCTTGACGAGTTCTTCTGAGCGGGAC TCTGGGGTTCGAAATGACCGACCAAGCGACGCCCAACCTGCCATCACGAGAT TTCGATTCCACCGCCGCCTTCTATGAAAGGTTGGGCTTCGGAATCGTTTTCCG GGACGCCGGCTGGATGATCCTCCAGCGCGGGGATCTCATGCTGGAGTTCTTC GCCCACCCCAACTTGTTTATTGCAGCTTATAATGGTTACAAATAAAGCAATA GCATCACAAATTTCACAAATAAAGCATTTTTTTCACTGCATTCTAGTTGTGGT TTGTCCAAACTCATCAATGTATCTTATCATGTCTGTATACCGTCGACCTCTAG CTAGAGCTTGGCGTAATCATGGTCATAGCTGTTTCCTGTGTGAAATTGTTATC CGCTCACAATTCCACACAACATACGAGCCGGAAGCATAAAGTGTAAAGCCT GGGGTGCCTAATGAGTGAGCTAACTCACATTAATTGCGTTGCGCTCACTGCC CGCTTTCCAGTCGGGAAACCTGTCGTGCCAGCTGCATTAATGAATCGGCCAA CGCGCGGGGAGAGGCGGTTTGCGTATTGGGCGCTCTTCCGCTTCCTCGCTCA CTGACTCGCTGCGCTCGGTCGTTCGGCTGCGGCGAGCGGTATCAGCTCACTC AAAGGCGGTAATACGGTTATCCACAGAATCAGGGGATAACGCAGGAAAGAA CATGTGAGCAAAAGGCCAGCAAAAGGCCAGGAACCGTAAAAAGGCCGCGTT GCTGGCGTTTTTCCATAGGCTCCGCCCCCCTGACGAGCATCACAAAAATCGA CGCTCAAGTCAGAGGTGGCGAAACCCGACAGGACTATAAAGATACCAGGCG TTTCCCCCTGGAAGCTCCCTCGTGCGCTCTCCTGTTCCGACCCTGCCGCTTAC CGGATACCTGTCCGCCTTTCTCCCTTCGGGAAGCGTGGCGCTTTCTCATAGCT CACGCTGTAGGTATCTCAGTTCGGTGTAGGTCGTTCGCTCCAAGCTGGGCTGT GTGCACGAACCCCCCGTTCAGCCCGACCGCTGCGCCTTATCCGGTAACTATC GTCTTGAGTCCAACCCGGTAAGACACGACTTATCGCCACTGGCAGCAGCCAC TGGTAACAGGATTAGCAGAGCGAGGTATGTAGGCGGTGCTACAGAGTTCTTG AAGTGGTGGCCTAACTACGGCTACACTAGAAGAACAGTATTTGGTATCTGCG CTCTGCTGAAGCCAGTTACCTTCGGAAAAAGAGTTGGTAGCTCTTGATCCGG CAAACAAACCACCGCTGGTAGCGGTTTTTTTGTTTGCAAGCAGCAGATTACG CGCAGAAAAAAAGGATCTCAAGAAGATCCTTTGATCTTTTCTACGGGGTCTG ACGCTCAGTGGAACGAAAACTCACGTTAAGGGATTTTGGTCATGAGATTATC 
AAAAAGGATCTTCACCTAGATCCTTTTAAATTAAAAATGAAGTTTTAAATCA ATCTAAAGTATATATGAGTAAACTTGGTCTGACAGTTACCAATGCTTAATCA GTGAGGCACCTATCTCAGCGATCTGTCTATTTCGTTCATCCATAGTTGCCTGA CTCCCCGTCGTGTAGATAACTACGATACGGGAGGGCTTACCATCTGGCCCCA GTGCTGCAATGATACCGCGAGACCCACGCTCACCGGCTCCAGATTTATCAGC AATAAACCAGCCAGCCGGAAGGGCCGAGCGCAGAAGTGGTCCTGCAACTTT ATCCGCCTCCATCCAGTCTATTAATTGTTGCCGGGAAGCTAGAGTAAGTAGT TCGCCAGTTAATAGTTTGCGCAACGTTGTTGCCATTGCTACAGGCATCGTGGT GTCACGCTCGTCGTTTGGTATGGCTTCATTCAGCTCCGGTTCCCAACGATCAA GGCGAGTTACATGATCCCCCATGTTGTGCAAAAAAGCGGTTAGCTCCTTCGG TCCTCCGATCGTTGTCAGAAGTAAGTTGGCCGCAGTGTTATCACTCATGGTTA TGGCAGCACTGCATAATTCTCTTACTGTCATGCCATCCGTAAGATGCTTTTCT GTGACTGGTGAGTACTCAACCAAGTCATTCTGAGAATAGTGTATGCGGCGAC CGAGTTGCTCTTGCCCGGCGTCAATACGGGATAATACCGCGCCACATAGCAG AACTTTAAAAGTGCTCATCATTGGAAAACGTTCTTCGGGGCGAAAACTCTCA AGGATCTTACCGCTGTTGAGATCCAGTTCGATGTAACCCACTCGTGCACCCA ACTGATCTTCAGCATCTTTTACTTTCACCAGCGTTTCTGGGTGAGCAAAAACA GGAAGGCAAAATGCCGCAAAAAAGGGAATAAGGGCGACACGGAAATGTTG AATACTCATACTCTTCCTTTTTCAATATTATTGAAGCATTTATCAGGGTTATT GTCTCATGAGCGGATACATATTTGAATGTATTTAGAAAAATAAACAAATAGG GGTTCCGCGCACATTTCCCCGAAAAGTGCCACCTGACGTC 